\title{
Association between maternal triglycerides and disturbed glucose metabolism in pregnancy
}

\author{
Daniel Eppel ${ }^{1} \cdot$ Michael Feichtinger $^{1,2} \cdot$ Tina Lindner $^{1,2} \cdot$ Grammata Kotzaeridi $^{1} \cdot$ Ingo Rosicky $^{1} \cdot$ \\ Guelen Yerlikaya-Schatten ${ }^{1} \cdot$ Wolfgang Eppel $^{1} \cdot$ Peter Husslein $^{1} \cdot$ Andrea Tura $^{3} \cdot$ Christian S. Göbl $^{1} \oplus$
}

Received: 23 September 2020 / Accepted: 20 November 2020 / Published online: 2 January 2021

(c) The Author(s) 2020

\begin{abstract}
Aims Dyslipidemia in pregnancy is associated with adverse pregnancy outcomes as elevated triglycerides might be considered as a risk factor for hyperglycemia and gestational diabetes. As only a few studies have addressed the association between maternal triglycerides and glucose metabolism, we aimed to explore the pathophysiologic associations of moderate hypertriglyceridemia and maternal glucose metabolism in pregnancy.

Methods Sixty-seven pregnant women received a detailed metabolic characterization at $12+0-22+6$ weeks of gestation by an extended 2h-75g OGTT (oral glucose tolerance test); with measurements of glucose, insulin and C-peptide at fasting and every $30 \mathrm{~min}$ after ingestion and assessment of triglycerides at fasting state. All examinations were repeated at 24+0-27+6 weeks of gestation.

Results Elevated triglycerides in early gestation were associated with insulin resistance and $\beta$-cell dysfunction. Mean glucose concentrations during the OGTT in early pregnancy were already higher in women with hypertriglyceridemia as compared to women with triglycerides in the normal range. A higher degree of insulin resistance and increased OGTT glucose levels were also observed when metabolic assessments were repeated between 24 and 28 weeks of gestation. Of note, elevated triglycerides at early gestation were associated with development of gestational diabetes by logistic regression (odds ratio: $1.16,95 \%$ CI: $1.03-1.34, p=0.022$ for an increase of $10 \mathrm{mg} / \mathrm{dl}$ ).

Conclusions Hypertriglyceridemia at the start of pregnancy is closely related to impaired insulin action and $\beta$-cell function. Women with hypertriglyceridemia have higher mean glucose levels in early- and mid-gestation. Pregnant women with elevated triglycerides in early pregnancy are at increased risk of developing gestational diabetes.
\end{abstract}

Keywords Hyperlipidemia $\cdot$ Insulin resistance $\cdot$ Pregnancy $\cdot \beta$-cell function

\section{Abbreviations}

BIS Basal insulin secretion

BMI Body mass index

CI Confidence intervals

This article belongs to the topical collection Pregnancy and Diabetes, managed by Antonio Secchi and Marina Scavini.

Electronic supplementary material The online version of this article (https://doi.org/10.1007/s00592-020-01644-z) contains supplementary material, which is available to authorised users.

Christian S. Göbl

christian.goebl@meduniwien.ac.at

1 Department of Obstetrics and Gynecology, Division of Obstetrics and Feto-Maternal Medicine, Medical University of Vienna, Vienna, Austria

2 Wunschbaby Institut Feichtinger, WIF, Vienna, Austria

3 Metabolic Unit, CNR Institute of Neuroscience, Padova, Italy

$\begin{array}{ll}\text { CP } & \text { C-peptide } \\ \text { GDM } & \text { Gestational diabetes mellitus } \\ \text { HTG } & \text { Moderate triglyceridemia } \\ \text { IQR } & \text { Interquartile range } \\ \text { NTG } & \text { Normal triglyceridemia } \\ \text { OGTT } & \text { Oral glucose tolerance test } \\ \text { OR } & \text { Odds ratio } \\ \text { QUICKI } & \text { Quantitative insulin sensitivity check index } \\ \text { TG } & \text { Triglycerides } \\ \text { TIS } & \text { Total insulin secretion }\end{array}$

\section{Background}

Gestational diabetes mellitus (GDM) is widely accepted as a major reason for adverse pregnancy outcomes including fetal macrosomia, cesarean section rate, neonatal hypoglycemia or the development of obesity in the newborns' 
later life [1-3]. In addition to the well-known effect of hyperglycemia, other metabolic characteristics could be of further importance for maternal and offspring health $[4,5]$. For example, previous studies indicated that alterations in maternal lipid metabolism were associated with adverse pregnancy outcomes, as elevated serum triglycerides concentrations were found to be associated with an increased risk of preeclampsia and the development of large for gestational age neonates [6]. As known from studies of non-pregnant women, maternal lipids may be intercorrelated with obesity and parameters of disturbed glucose metabolism (especially insulin resistance), which are commonly known risk factors for the development of hyperglycemia [7]. Therefore, increased maternal lipid content could be regarded as a potential risk factor for hyperglycemia in pregnancy as well [4]. However, only a few studies have addressed the possible cross-link between maternal triglyceride concentrations and parameters of glucose metabolism during gestation, and consequently, the evidence for possible clinical implications of increased lipid concentrations in pregnancy is sparse.

Therefore, this study aims to assess the pathophysiologic characteristics of subclinical hyperlipidemia in early pregnancy. In particular, associations between maternal triglycerides and parameters of glucose metabolism (including insulin sensitivity, insulin secretion and $\beta$-cell function) are described, and their possible association with the consecutive development of GDM is assessed as a secondary objective.

\section{Participants and methods}

\section{Study design and participants}

The present explorative study aimed at identifying risk factors for glucometabolic disorders in pregnancy as published in detail elsewhere [8]. In short, sixty-seven pregnant women received a detailed metabolic characterization at the first study visit (median gestational age: 18.3 weeks, IQR: $16.0-20.3$ weeks) by an extended $2 \mathrm{~h}-75 \mathrm{~g}$ OGTT (with measurements of glucose, insulin and C-peptide at fasting and every 30 min after ingestion), and laboratory assessment of triglycerides (TG) at fasting state, as well as anthropometric measurements. For descriptive purposes, the study participants were classified according to their triglycerides concentration in early gestation: normal triglycerides (NTG) if TG was equal or below 150 $\mathrm{mg} / \mathrm{dl}$ and moderate hypertriglyceridemia (HTG) if TG exceeded $150 \mathrm{mg} / \mathrm{dl}$, according to several guidelines [9, 10]. All examinations were repeated at $24+0-27+6$ weeks of gestation. GDM was diagnosed by using the IADPSG criteria [11]. Patients with pre-existing diabetes were not included in this study. All laboratory parameters were measured according to the standard laboratory methods at our certified Department of Medical and Chemical Laboratory Diagnostics (http://www.kimcl.at). The study was approved by the Ethics Committee of the Medical University of Vienna and performed in accordance with the Declaration of Helsinki. All participants gave written informed consent.

\section{Calculation of parameters of glucose homeostasis}

The quantitative insulin sensitivity check index (QUICKI) was used to assess insulin sensitivity at fasting state [12]. Moreover, we used dynamic indices of insulin action from OGTT data such as the composite index (ISI-comp) as well as the most recently developed PREDIM index to quantify whole- body insulin action [13, 14]. Overall insulin response to glucose was calculated by use of a modified insulinogenic index (AUC-insulin/AUC-glucose 0-120) [15]. The extent to which the pancreatic $\beta$-cells can adapt to impaired insulin sensitivity was examined by the ISSI- 2 index (sometimes called the oral disposition index) as ISI-comp $\times$ AUC-insulin/AUC-glucose 0-120. In addition, basal insulin secretion (BIS) and total insulin secretion rate (TIS) were assessed by mathematical modelling from insulin and C-peptide data according to [16].

\section{Statistical analysis}

Categorical variables were summarized by counts and percentages and compared by Fisher's exact test. Continuous scaled variables were summarized by means and standard deviations and compared by Student's $t$ test. Skewed distributed parameters were summarized by mean and interquartile range and compared by an exact Wilcoxon rank-sum test. Analysis of covariance (ANCOVA) was used to adjust for possible confounders like differences in maternal BMI (body mass index). Bivariate associations between ordinal and metric scaled variables were assessed by Spearman's correlation coefficient.

Statistical analysis was performed by R (V 3.5.3) and contributing packages. The two-sided significance level was set to 0.05 . However, p-values were interpreted in an explorative manner, and therefore, there was no further adjustment for multiplicity if not otherwise indicated in the manuscript.

\section{Results}

Twenty-seven patients with moderate hypertriglyceridemia were identified. As shown in Table 1 elevated triglycerides in early gestation were associated with impaired insulin 
sensitivity as expressed by significantly lower index levels of PREDIM as well as higher BMI, and the differences in insulin action between the subgroups remained significant in multivariable analysis after adjustment for pregestational BMI and age (adjusted mean difference for PREDIM after log transformation: $0.15,95 \% \mathrm{CI}$ : $0.02-0.29, p=0.028$ ). The higher insulin resistance observed in women with hypertriglyceridemia was accompanied by higher basal and total insulin secretion, although lower ISSI-2 index indicated insufficient compensation of insulin secretion for the observed degree of insulin resistance (Fig. 1). As a consequence, already at the beginning of pregnancy, mean glucose concentrations during the OGTT were increased in women with moderate hypertriglyceridemia, as compared to women with triglycerides in the normal range. Higher degrees of insulin resistance and increased OGTT glucose levels were also observed when metabolic assessments were repeated between 24 and 28 weeks of gestation (log transformed PREDIM $\left[\mathrm{mg} \mathrm{kg}^{-1} \mathrm{~min}^{-1}\right.$ ]: $1.09 \pm 0.35$ vs. $0.84 \pm 0.44, p=0.032$; mean glucose $[\mathrm{mg} / \mathrm{dl}]: 105 \pm 13$ vs. $121 \pm 25, p=0.011)$. The median increase in triglyceride concentrations from first to second visit was $41 \mathrm{mg} / \mathrm{dl}$ (IQR: $12-79 \mathrm{mg} / \mathrm{dl}$ ). A detailed analysis of the correlation between maternal triglycerides and glucose metabolism at the second visit suggested that parameters of glucose disposal were rather related to baseline triglycerides than to the change in triglyceride levels between the two visits (Table 2). Six cases of GDM occurred in this study. The association between development of GDM and triglycerides as well as other lipids parameters is provided in supplemental material (Table S1). Although the limited sample size has to be considered for interpreting these results, maternal triglycerides at early gestation were associated with development of GDM (OR: 1.16, 95\%CI: $1.03-1.34, p=0.022$ for an increase of $10 \mathrm{mg} / \mathrm{dl}$ ), whereas this association was not observed for total cholesterol, HDL cholesterol and LDL cholesterol.
Table 1: Glucometabolic parameters of the study sample at early gestation. Patients with and without subtle hypertriglyceridemia (NTG vs. HTG) at $12+0-22+6$ weeks of gestation.

\begin{tabular}{|c|c|c|c|}
\hline & NTG & HTG & p-value \\
\hline Age (years) & $29.0 \pm 5.1$ & $30.9 \pm 5.1$ & 0.129 \\
\hline BMIPG $\left(\mathrm{kg} / \mathrm{m}^{2}\right)$ & $22.2(20.5-24.0)$ & $23.5(22.2-27.6)$ & 0.035 \\
\hline BMIV1 $\left(\mathrm{kg} / \mathrm{m}^{2}\right)$ & $24.2(22.0-26.4)$ & $25.5(24.1-29.3)$ & 0.042 \\
\hline Parity & $0.0(0.0-1.0)$ & $0.0(0.0-1.0)$ & 0.620 \\
\hline GDM previous pregnancy & $3(7.5 \%)$ & $2(7.4 \%)$ & 1.000 \\
\hline TG (mg/dl) & $117.7 \pm 20.8$ & $217.8 \pm 48.6$ & $<0.001$ \\
\hline $\mathrm{TC}(\mathrm{mg} / \mathrm{dl})$ & $214.0 \pm 39.8$ & $231.6 \pm 40.1$ & 0.082 \\
\hline LDL-C (mg/dl) & $112.6 \pm 31.1$ & $117.5 \pm 32.7$ & 0.546 \\
\hline HDL-C (mg/dl) & $80.5(65.0-88.8)$ & $66.0(59.5-77.5)$ & 0.067 \\
\hline G0 (mg/dl) & $75.0(72.0-78.0)$ & $78.0(73.0-82.0)$ & 0.126 \\
\hline G-mean (mg/dl) & $96.7(84.3-105.3)$ & $112.0(96.8-131.3)$ & 0.003 \\
\hline $\mathrm{I} 0(\mu \mathrm{U} / \mathrm{ml})$ & $6.17(3.33-8.55)$ & $7.25(5.09-14.10)$ & 0.068 \\
\hline I-mean $(\mu \mathrm{U} / \mathrm{ml})$ & $33.0(19.1-49.7)$ & $54.3(27.0-91.1)$ & 0.090 \\
\hline CP0 (ng/ml) & $1.50(1.20-1.73)$ & $1.70(1.40-2.50)$ & 0.040 \\
\hline CP-mean (ng/ml) & $5.33(4.22-6.63)$ & $6.86(4.90-8.92)$ & 0.048 \\
\hline QUICKI (dimensionless) & $0.16(0.15-0.18)$ & $0.16(0.14-0.17)$ & 0.074 \\
\hline ISI-comp (dimensionless) & $8.51(5.91-15.10)$ & $6.13(3.43-9.90)$ & 0.045 \\
\hline $\operatorname{PREDIM}\left(\mathrm{mg} \mathrm{kg}^{-1} \min ^{-1}\right)$ & $3.63(2.91-4.41)$ & $2.67(1.80-3.39)$ & 0.006 \\
\hline AUC-I/AUC-G [0-120 min] $(\mu \mathrm{U} / \mathrm{mg})$ & $41.5 \pm 24.9$ & $50.6 \pm 31.6$ & 0.318 \\
\hline 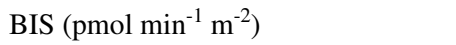 & $70.5 \pm 23.3$ & $87.0 \pm 34.4$ & 0.035 \\
\hline TIS $\left(\mathrm{nmol} / \mathrm{m}^{-2}\right)$ & $39.7 \pm 13.4$ & $48.3 \pm 18.7$ & 0.046 \\
\hline ISSI-2 (dimensionless) & $2.93(2.30-3.73)$ & $2.58(1.86-3.12)$ & 0.037 \\
\hline
\end{tabular}

Data are expressed as mean \pm standard deviation or median and interquartile range. Values are given for pregestational body mass index (BMIPG), body mass index at visit 1 (BMIV1), triglycerides (TG), total cholesterol (TC), LDL cholesterol (LDL-C), HDL cholesterol (HDL-C), glucose (G), insulin (I) and C-peptide (CP) at fasting and as mean values during the OGTT.

QUICKI quantitative insulin sensitivity check index; ISI-comp, insulin sensitivity composite index; PREDIM predicted M; overall insulin secretion (AUC-I/AUC-G [0-120 min]); BIS basal insulin secretion rate; $T I S$ total insulin secretion rate from C-peptide; ISSI-2, oral disposition index 
a

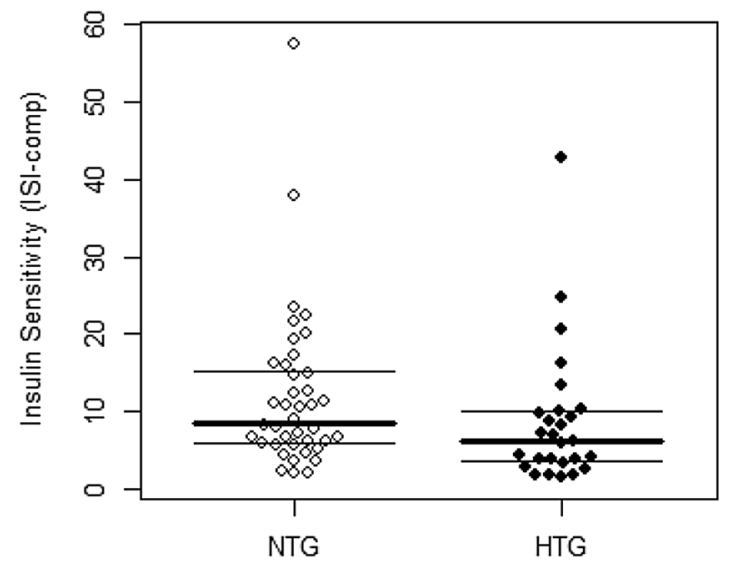

C

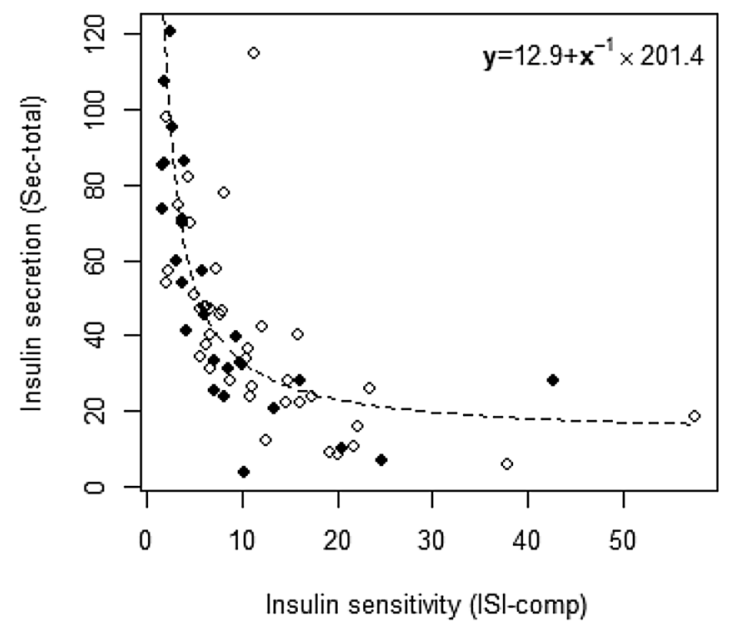

Figure 1: Bee swarm plots representing comparisons of insulin action (a) and insulin secretion (b), the association between insulin sensitivity and secretion showing the hyperbolic regression line (c) and the

\section{Discussion}

This study aimed at investigating pathophysiological patterns of glucose metabolism in association with fasting triglyceride levels. We found that moderate hypertriglyceridemia at the start of pregnancy was related to impaired insulin action and $\beta$-cell function. This was accompanied by elevated glucose levels at mid-gestation. Consequently, increased serum triglycerides were associated with a higher risk for the development of GDM.

In the non-pregnant state, increased levels of triglycerides are a well-known characteristic of dyslipidemia associated with insulin resistance and are considered as an important risk factor for the development of diabetes and cardiovascular disease [17][18][19]. However, in the last decades, research interest in dyslipidemia in pregnancy was low, although more recently the spotlight shifted. It is well known b

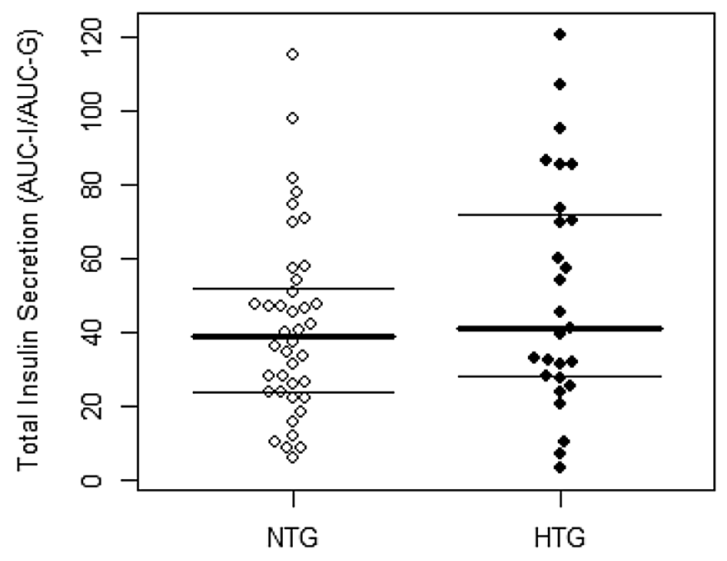

d

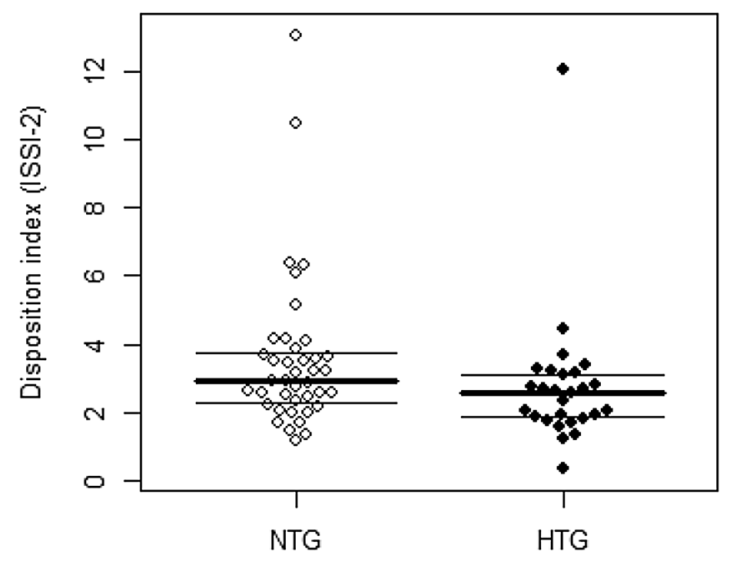

comparison of the oral disposition index (d) at early gestation. NTG, normal triglycerides; HTG, hypertriglyceridemia.

that during course of physiologic gestation, plasma triglycerides concentrations increase due to impaired removal by lipolytic enzymes (such as lipoprotein lipase) [20]. This process may be further triggered by overweight or obesity, as a recent study published by Bozkurt et al. found that obesity was associated with abnormal lipid constellation and mainly elevated triglycerides concentration, maybe as a consequence of impaired insulin action in these patients [21]. This is in line with the results of our study, demonstrating decreased indices of insulin sensitivity in mothers with moderate hypertriglyceridemia even at early pregnancy, although we found that the association between increased triglycerides and impaired insulin action was independent of BMI, suggesting an association between maternal triglycerides and glucose metabolism which is independent of overweight or obesity status. Moreover, we found that parameters of glucose metabolism at the second visit were 
Table 2: Association of glucometabolic parameters at V2 (24-28 weeks of gestation) with triglyceride levels at V1 (baseline visit) and their change (V2 - V1)

\begin{tabular}{|c|c|c|c|c|}
\hline \multirow[t]{2}{*}{ V2 } & \multicolumn{2}{|c|}{$\begin{array}{l}\text { Association with base- } \\
\text { line TG (V1) }\end{array}$} & \multicolumn{2}{|c|}{$\begin{array}{l}\text { Association with } \\
\text { change of TG (V2- } \\
\text { V1) }\end{array}$} \\
\hline & rho & $p$-value & rho & $p$-value \\
\hline G0 & 0.19 & N.S. & 0.14 & N.S. \\
\hline G-mean & 0.42 & $<0.001$ & 0.03 & N.S. \\
\hline I0 & 0.21 & N.S. & 0.09 & N.S. \\
\hline I-mean & 0.30 & 0.023 & -0.00 & N.S. \\
\hline CP0 & 0.55 & $<0.001$ & 0.14 & N.S. \\
\hline CP-mean & 0.43 & $<0.001$ & 0.19 & N.S. \\
\hline QUICKI & -0.21 & N.S. & -0.10 & N.S. \\
\hline ISI-comp & -0.29 & 0.026 & -0.05 & N.S. \\
\hline PREDIM & -0.41 & 0.001 & -0.08 & N.S. \\
\hline AUC-I/AUC-G & 0.14 & N.S. & -0.05 & N.S. \\
\hline BIS & 0.55 & $<0.001$ & 0.09 & N.S. \\
\hline TIS & 0.40 & 0.002 & 0.15 & N.S. \\
\hline ISSI-2 & -0.21 & N.S. & -0.03 & N.S. \\
\hline
\end{tabular}

Data are expressed as Spearman's correlation coefficient (rho). Values are given for glucose $(\mathrm{G})$, insulin (I) and C-peptide (CP) at fasting and as mean values during the OGTT.

QUICKI quantitative insulin sensitivity check index; ISI-comp insulin sensitivity composite index; PREDIM predicted M; overall insulin secretion (AUC-I/AUC-G [0-120 min]); BIS basal insulin secretion rate; TIS total insulin secretion rate from C-peptide; ISSI-2, oral disposition index

rather related to baseline triglycerides than to the change in triglyceride levels between the two visits.

Some studies have investigated the association between lipid levels and glucose metabolism in pregnancy: SanchezVera et al. assessed differences in maternal lipid content in women who developed GDM as compared to normal glucose tolerant control women and observed elevated levels of triglycerides in women with GDM in the first trimester, already before GDM was diagnosed [22]. In another study, Bao et al. characterized longitudinal changes in lipid profiles throughout pregnancy and examined the association of plasma lipid levels with risk of GDM. The authors concluded that higher triglyceride levels in early and midpregnancy were significantly associated with greater risk of GDM manifestation [23]. Furthermore, Enquobahrie et al. examined maternal lipid levels in early pregnancy and the risk of GDM and observed that elevated triglycerides were positively associated with GDM [24]. Although the number of GDM cases is small in our study, what is a limitation of our work, the above-mentioned three studies are in accordance with our results, indicating that pregnant women with elevated triglycerides in early pregnancy are at increased risk for GDM. This association can be explained by a higher degree of insulin resistance, which is not adequately compensated by increased $\beta$-cell secretion. We observed not only impaired insulin sensitivity, but also lower ISSI-2 levels indicating insufficient compensation by the $\beta$-cells. Thus, elevated triglycerides at early gestation may be regarded as a potential biomarker for prediabetic disorders that will lead to hyperglycemia when pregnancy progresses and the physiologic environment changes. Although the establishment of early risk assessment tools is challenging due to the heterogeneity of the disease [25], a recent study by Benhalima et $a l$. found that the inclusion of biomarkers such as triglycerides improves the accuracy of risk assessment models to predict the risk for the development of GDM at the start of pregnancy, as also underlined by the data of our study [26]. Of note, we observed no association between cholesterol (LDL, HDL or total cholesterol) with GDM development.

To our knowledge, this is the first study investigating the cross-link between elevated triglycerides and parameters of glucose metabolism, and we identified moderate hypertriglyceridemia as a potential risk factor for glycemic disorders in pregnancy. Although this aspect is novel, our study was not designed to assess implications for the newborns. This may be of additional interest, as maternal triglycerides are recognized as an important and physiologic fuel substrate for the growing fetus, but with a growing body of evidence proposing that increased maternal triglycerides correlate with excess fetal growth. Of note, maternal triglycerides have been associated with increased rates of large for gestational age newborns [27][28][29]. Moreover, associations with hypertension and preeclampsia were described indicating that further research is necessary to assess the potential role of elevated triglycerides as a risk factor for mothers and children [30].

Distinguishing independent contributions of prepregnancy obesity, pre-pregnancy insulin resistance or maternal lipid levels to impaired insulin resistance are challenging. We conclude that even moderately elevated triglycerides at the beginning of pregnancy are associated with impaired insulin action and $\beta$-cell dysfunction and hence with a potential risk factor for GDM development.

\section{Conclusion}

This study investigated pathophysiological patterns of maternal glucose metabolism during pregnancy in association with fasting triglyceride levels. Hypertriglyceridemia at the start of pregnancy was closely related to impaired insulin action and $\beta$-cell function. Women with hypertriglyceridemia had higher mean glucose levels in early- and mid-gestation. Consequently, increased serum triglycerides were associated with a higher risk for the development of GDM. 
Acknowledgements The authors thank all team members and participants in the study.

Authors' contributions CSG and MF conceived the study. Data assessment and patient recruitment were performed by CSG, MF and IR. Calculations and data interpretation were performed by CSG, DE and AT. Statistical analysis was performed by CSG. CSG prepared tables and figures. The manuscript was written by CSG and DE. AT, WE, IR, $\mathrm{PH}, \mathrm{GK}, \mathrm{TL}$ and GYS critically revised the manuscript. All authors reviewed and edited the final draft of the manuscript.

Funding Open access funding provided by Medical University of Vienna. The study was awarded a grant of the AGFMM (Arbeitsgruppe für Geburtshilfe und Feto-maternale Medizin) of the Austrian Association of Obstetrics and Gynecology to CSG. The funding source was not involved in study design; in the collection, analysis and interpretation of data; in the writing of the manuscript; and in the decision to submit the article for publication.

Availability of data and materials The datasets used and analyzed during the current study are available from the corresponding author on reasonable request.

\section{Compliance with Ethical Standards}

Conflict of interest The authors declare that they have no competing interests.

\section{Consent for publication Not applicable.}

Ethics approval and consent to participate The study was approved by the Ethics Committee of the Medical University of Vienna and performed in accordance with the Declaration of Helsinki. All participants gave written informed consent.

Open Access This article is licensed under a Creative Commons Attribution 4.0 International License, which permits use, sharing, adaptation, distribution and reproduction in any medium or format, as long as you give appropriate credit to the original author(s) and the source, provide a link to the Creative Commons licence, and indicate if changes were made. The images or other third party material in this article are included in the article's Creative Commons licence, unless indicated otherwise in a credit line to the material. If material is not included in the article's Creative Commons licence and your intended use is not permitted by statutory regulation or exceeds the permitted use, you will need to obtain permission directly from the copyright holder. To view a copy of this licence, visit http://creativecommons.org/licenses/by/4.0/.

\section{References}

1. HAPO Study Cooperative Research Group, Metzger BE, Lowe LP, Dyer AR et al (2008) Hyperglycemia and adverse pregnancy outcomes. N Engl J Med 358(19):1991-2002

2. Scavini M, Secchi A (2019) Diabetes in pregnancy. Acta Diabetol 56(7):719-21

3. Shokry E, Marchioro L, Uhl O et al (2019) Transgenerational cycle of obesity and diabetes: investigating possible metabolic precursors in cord blood from the PREOBE study. Acta Diabetol 56(9):1073-82
4. Catalano PM, Shankar K (2017) Obesity and pregnancy: mechanisms of short term and long term adverse consequences for mother and child. BMJ 8(356):j1

5. Agha-Jaffar R, Oliver N, Johnston D (2016) Gestational diabetes mellitus: does an effective prevention strategy exist? Nat Rev Endocrinol 12(9):533-46

6. Wiznitzer A, Mayer A, Novack V et al (2009) Association of lipid levels during gestation with preeclampsia and gestational diabetes mellitus: a population-based study. Am J Obstet Gynecol 201(5):482.e1-8

7. Goldberg IJ (2001) Clinical review 124: diabetic dyslipidemia: causes and consequences. J Clin Endocrinol Metab 86(3):965-71

8. Stopp T, Feichtinger M, Rosicky I et al (2020) Novel indices of glucose homeostasis derived from principal component analysis: application for metabolic assessment in pregnancy. J Diabet Res 2020:4950584

9. Grundy SM, Stone NJ, Bailey AL et al (2019) 2018 AHA/ACC/ AACVPR/AAPA/ABC/ACPM/ADA/AGS/APhA/ASPC/NLA/ PCNA guideline on the management of blood cholesterol. J Am Coll Cardiol 73(24):e285-350

10. Mach F, Baigent C, Catapano AL et al (2020) 2019 ESC/EAS Guidelines for the management of dyslipidaemias: lipid modification to reduce cardiovascular risk. Eur Heart J 41(1):111-88

11. International Association of Diabetes and Pregnancy Study Groups Consensus Panel (2010) International association of diabetes and pregnancy study groups recommendations on the diagnosis and classification of hyperglycemia in pregnancy. Diabet Care 33(3):676-82

12. Katz A, Nambi SS, Mather K et al (2000) Quantitative insulin sensitivity check index: a simple, accurate method for assessing insulin sensitivity in humans. J Clin Endocrinol Metab 85(7):2402-10

13. Matsuda M, DeFronzo RA (1999) Insulin sensitivity indices obtained from oral glucose tolerance testing: comparison with the euglycemic insulin clamp. Diabet Care 22(9):1462-70

14. Tura A, Chemello G, Szendroedi J et al (2018) Prediction of clamp-derived insulin sensitivity from the oral glucose insulin sensitivity index. Diabetologia 61(5):1135-41

15. Tura A, Kautzky-Willer A, Pacini G (2006) Insulinogenic indices from insulin and C-peptide: comparison of betacell function from OGTT and IVGTT. Diabet Res Clin Pract 72(3):298-301

16. Mari A, Tura A, Gastaldelli A, Ferrannini E (2002) Assessing insulin secretion by modeling in multiple-meal tests: role of potentiation. Diabetes 51(Suppl 1):S221-226

17. Bansal S, Buring JE, Rifai N (2007) Fasting compared with nonfasting triglycerides and risk of cardiovascular events in women. JAMA 298(3):309-16

18. Freiberg JJ, Tybjaerg-Hansen A, Jensen JS, Nordestgaard BG (2008) Nonfasting triglycerides and risk of ischemic stroke in the general population. JAMA 300(18):2142-52

19. Hjellvik V, Sakshaug S, Strøm H (2012) Body mass index, triglycerides, glucose, and blood pressure as predictors of type 2 diabetes in a middle-aged Norwegian cohort of men and women. Clin Epidemiol 4:213-24

20. Kinoshita T, Shirai K, Itoh M (2003) The level of pre-heparin serum lipoprotein lipase mass at different stages of pregnancy. Clinica Chimica Acta 337(1-2):153-6

21. Bozkurt L, Gobl CS, Hormayer A-T (2016) The impact of preconceptional obesity on trajectories of maternal lipids during gestation. Sci Rep 20(6):29971

22. Sanchez-Vera I, Bonet B, Viana M et al (2007) Changes in plasma lipids and increased low-density lipoprotein susceptibility to oxidation in pregnancies complicated by gestational diabetes: consequences of obesity. Metabolism 56(11):1527-33 
23. Bao W, Dar S, Zhu Y et al (2018) Plasma concentrations of lipids during pregnancy and the risk of gestational diabetes mellitus: a longitudinal study. J Diabet 10(6):487-95

24. Enquobahrie DA, Williams MA, Qiu C (2005) Early pregnancy lipid concentrations and the risk of gestational diabetes mellitus. Diabetes Res Clin Pract 70(2):134-42

25. Huvinen E, Eriksson JG, Stach-Lempinen B (2018) Heterogeneity of gestational diabetes (GDM) and challenges in developing a GDM risk score. Acta Diabet 55(12):1251-9

26. Benhalima K, Van Crombrugge P, Moyson C et al (2020) Estimating the risk of gestational diabetes mellitus based on the 2013 WHO criteria: a prediction model based on clinical and biochemical variables in early pregnancy. Acta Diabet 57(6):661-671

27. Vrijkotte TGM, Krukziener N, HuttenTwickler BA (2012) Maternal lipid profile during early pregnancy and pregnancy complications and outcomes: the ABCD study. J Clin Endocrinol Metab 97(11):3917-25
28. Wang J, Moore D, Subramanian A et al (2018) Gestational dyslipidaemia and adverse birthweight outcomes: a systematic review and meta-analysis. Obes Rev 19(9):1256-68

29. Samsuddin S, Arumugam PA, Md Amin MS et al (2020) Maternal lipids are associated with newborn adiposity, independent of GDM status, obesity and insulin resistance: a prospective observational cohort study. BJOG 127(4):490-9

30. Gallos ID, Sivakumar K, Kilby MD (2013) Pre-eclampsia is associated with, and preceded by, hypertriglyceridaemia: a metaanalysis. BJOG 120(11):1321-32

Publisher's Note Springer Nature remains neutral with regard to jurisdictional claims in published maps and institutional affiliations. 